\title{
Abstract \\ Massive outbreak of measles in 2013, an experience in Teaching Hospital Anuradhapura \\ Ganegama $\mathrm{RM}^{1 *}$, Malawara Arachchi $\mathrm{NS}^{1}$, Senevirathne $\mathrm{SS}^{1}$, Karunamuni $\mathrm{N}^{1}$, Agampodi $\mathrm{SB}^{1}$, Dahanavaka $\mathrm{N}^{2}$, Prasanna $\mathrm{P}^{2}$, Pahalagamage $\mathrm{S}^{3}$ \\ ${ }^{1}$ Department of Community Medicine, Faculty of Medicine and Allied Sciences, Rajarata University of Sri Lanka \\ ${ }^{2}$ Department of Medicine, Faculty of Medicine and Allied Sciences, Rajarata University of Sri Lanka ${ }^{3}$ University Medical Unit, Teaching Hospital Anuradhapura
}

\begin{abstract}
\section{Background}

Measles vaccine was introduced to the expanded program of immunization (EPI) of Sri Lanka in August 1984 as a single dose and extended to a double dose vaccine following the measles outbreak in 1999-2000. After this intervention the incidence of the measles was under control until 2013. Purpose of this study was to describe the measles outbreak in 2013.

\section{Methods}

Spatio-temporal distribution of the island wide outbreak was analysed using surveillance data from Weekly Epidemiological Reports published by the Epidemiology Unit of the Sri Lanka from 2012 to 2014. A prospective study was carried out in the university medical unit of teaching hospital, Anuradhapura from October 2013 to describe the clinical-epidemiological profile of measles patients.

\section{Results}

There was a clear geographical variation in measles incidence in Sri Lanka during the 2013 outbreak. Increase of measles incidence was detected since the latter part of 2012. From 2013 January, 101 patients suspected with measles were admitted to the Teaching Hospital Anuradhapura (THA). Until June 2013, all suspected cases were less than 12 months of age. After June 2013, more adults were admitted. We recruited 32 patients for the clinical study, of whom, most showed typical clinical features except Koplik spots which were there in only 8 patients. Features suggestive of pneumonia were observed among $30(90.9 \%)$ patients. Two patients $(6.3 \%)$ with severe pneumonia received ICU care due to respiratory difficulties. Out of 32 patients, $15(45.5 \%)$ had prior immunization for measles, 2 reported that they never had a measles immunization and 16 were unsure about their immunization status. Of those reported as previously immunized, 11 (73.3\%) belonged to the age group 12-29 years.

\section{Conclusions}

This outbreak in Anuradhapura was started among infants. Sudden increase of susceptible cohort due to change in immunization schedule could be a trigger factor for this outbreak.
\end{abstract}

Key words: Measles; Outbreak; Anuradhapura; Sri Lanka

Copyright: $\odot 2015$ Ganegama RM et al. This is an open access article distributed under the Creative Commons Attribution License, which permits unrestricted use, distribution, and reproduction in any medium, provided the original work is properly cited.

* Correspondence : ranjanganegama@gmail.com

Cite this abstract as: Ganegama $\mathrm{RM}^{1}$, Malawara Arachchi $\mathrm{NS}^{1}$, Senevirathne $\mathrm{SS}^{1}$, Karunamuni $\mathrm{N}^{1}$, Agampodi $\mathrm{SB}^{1}$, Dahanayaka $\mathrm{N}^{2}$, Prasanna $\mathrm{P}^{2}$, Pahalagamage $\mathrm{S}^{3}$. Massive outbreak of measles in 2013, an experience in Teaching Hospital Anuradhapura. Anuradhapura Medical Journal 2015;9 (2Supp):S41.

DOI: http://dx.doi.org/10.4038/amj.v9i2Supp.7590 
